\title{
ONTOGENIA DE LA FORMA EN LOS SERES VIVOS*
}

\author{
Miguel Concha \\ Universidad de Chile
}

\begin{abstract}
Resumen: La forma en los seres vivos se origina en las dimensiones temporales de la ontogenia y la filogenia, utilizando estrategias de tipo determinístico y regulativo. En la ontogenia de los animales, la forma se expresa inicialmente
\end{abstract}

Miguel Concha (Montevideo, 1966) es médico-cirujano y doctor en Ciencias Biomédicas de la Universidad de Chile. Profesor titular de anatomía y biología del desarrollo, Facultad de Medicina, Universidad de Chile. Es director del Laboratorio de Estudios Ontogénicos (LEO), en el cual investiga los mecanismos genéticos y morfogenéticos que subyacen a la generación de forma, estructura y organización funcional durante el desarrollo ontogénico. Investigador y miembro fundador del Instituto de Neurociencias Biomédicas de la Universidad de Chile. Dirección electrónica: mconcha@med.uchile.cl.

Quiero agradecer a Leonel Muñoz, Iskra Signore, Eduardo Pulgar y Eloísa Llorca por sus comentarios e ideas en versiones preliminares de este texto, y a todos los miembros del Laboratorio de Estudios Ontogénicos (LEO) por su apoyo. Nuestra investigación científica es financiada en la actualidad por proyectos de Fondecyt (1120558), la Iniciativa Científica Milenio (P09-015-F), y el Howard Hughes Medical Institute.

* Texto de la conferencia presentada en el ciclo para estudiantes universitarios "Formas de la realidad", organizado por el CEP el 22 de septiembre de 2011. Véase en esta misma edición la conferencia de Marcelo Boeri sobre la noción de forma en Platón y Aristóteles. Próximamente se publicarán en Estudios Públicos las conferencias de Enrique Barros B. y Luis Izquierdo sobre la forma en el derecho y en la arquitectura, respectivamente. (N. del E.) 
en las células, las que funcionan como unidades morfogenéticas primordiales o ladrillos generadores de forma, y a partir de ellas se transmite a niveles de organización biológica más complejos tales como tejidos y órganos, para finalmente moldear al organismo completo. El proceso generador de forma no sólo depende de la acción de genes y de su interacción con el medio ambiente, sino que además se rige por los principios generales de la física y las matemáticas, una idea inspirada en el legado de Sir D'Arcy Wentworth Thompson en su libro titulado On Growth and Form (1917).

Palabras clave: forma, morfogénesis, biología, ontogenia.

\section{ONTOGENY OF THE FORM OF LIVING BEINGS}

Abstract: The form of living beings has its origin in the temporal dimensions of ontogeny and phylogeny and is the result of deterministic and regulative strategies. In animal ontogeny, form is initially expressed in the cells, which work as primary morphogenetic units or form building blocks, and it is then transmitted from them to more complex levels of biological organization such as tissue and organs, to finally mold the entire organism. The generation of form depends not only on the action of genes and their interaction with the environment, but is also governed by the general principles of physics and mathematics, an idea inspired by the legacy of Sir D'Arcy Wentworth Thompson in his book entitled On Growth and Form (1917).

Keywords: form, morphogenesis, biology, ontogeny. 
$\mathrm{E}_{1}$

concepto de "forma" ha sido abordado por las ciencias biológicas desde distintas perspectivas del conocimiento. En un sentido primordial, forma hace referencia a la figura espacial o geométrica que describe a un ser vivo o a sus partes. Esta idea es ilustrada de manera muy gráfica en uno de los dibujos más difundidos del pintor renacentista Leonardo da Vinci (1452-1519), El Hombre de Vitruvio (1487) ${ }^{1}$. En esta obra, las dimensiones y proporciones de la figura humana se delimitan por medio de figuras geométricas, simbolizando el papel central de las matemáticas y la física en el entendimiento de los procesos biológicos que dan origen a la forma en los seres vivos. En un sentido más abstracto y siguiendo el pensamiento filosófico aristotélico, la forma tiene la propiedad de definir al ser vivo como un ser "real" en sus diversos grados de determinación, ya sustancial, ya accidental. Un "ser vivo" posee forma si contiene el principio activo que le otorga tal identidad, siendo la naturaleza de este principio el foco central de debate entre las visiones preformacionista y epigenetista ${ }^{2}$. En la primera, la forma biológica se manifiesta y desarrolla como un simple crecimiento de un germen de origen divino, mientras que en la segunda la forma se genera a sí misma a través de la interacción de elementos activos y pasivos otorgados por los progenitores. Hoy en día, la disciplina de la biología del desarrollo ${ }^{3}$ ha logrado rescatar elementos de ambas visiones y propone que ellas representan dos caras de un mismo proceso.

\section{Forma y tiempo en la biología}

El concepto de "forma biológica" está íntimamente ligado a la noción de "tiempo biológico". La forma en un ser vivo se adquiere a través de procesos dinámicos que acontecen en dos dimensiones temporales de naturaleza diversa, conocidas como ontogenia y filogenia. Para

1 Obra inspirada en un relato de Marco Vitrubio, arquitecto de Julio César, encontrado en los Diez Libros de Arquitectura. En el relato se hace mención al cuerpo de un hombre perfectamente apoyado dentro de un círculo (el símbolo divino) y un cuadrado (el símbolo terrenal), como una interpretación geométrica de que el hombre representa un microcosmos.

2 Una explicación didáctica de este debate se puede encontrar en $\mathrm{Ma}$ king Visible Embryos: http://www.hps.cam.ac.uk/visibleembryos/index.html.

3 Rama de la biología que estudia los procesos mediante los cuales los organismos crecen y se desarrollan. 
ilustrar este concepto utilizaremos el Tangram, un rompecabezas chino que se basa en formar siluetas ordenando siete piezas geométricas o tans sobre una superficie plana, sin solaparlas ${ }^{4}$. Este juego universal que ha entretenido a niños y adultos durante siglos permite ligar, de manera lúdica, la manipulación concreta de materiales con la formación de ideas abstractas. Además, resalta el hecho de que tanto la ontogenia como la filogenia reutilizan elementos o características previamente existentes para organizar y generar los cambios de forma.

En la Figura $\mathrm{N}^{\mathrm{o}} 1$ observamos dos secuencias de Tangram que representan las dimensiones temporales de la ontogenia y la filogenia. La ontogenia contempla el desarrollo de un organismo desde su concepción hasta su muerte, lo cual puede comprender sólo unos días en muchos insectos, décadas en los seres humanos, y cientos de años para el caso de muchas plantas. La filogenia, por otro lado, contempla las transformaciones que las especies experimentan durante su evolución, en una escala de miles a millones de años. Aun cuando la ontogenia y la filogenia acontecen en escalas temporales muy disímiles y poseen diferencias en su "operar y consecuencias", ellas exhiben sorprendentes similitudes. Por ejemplo, ambas se basan en los principios de “conservación de la organización" y "adaptación al cambio". Un ser vivo se define como tal en la medida que conserve su organización y que las transformaciones que experimente sean sólo adaptaciones al medio en el cual se desarrolla ${ }^{6}$. Cuando la organización de un ser vivo se altera, éste deja de vivir en la dimensión ontogénica. Si hablamos de evolución, una alteración de la organización significa la extinción de la especie.

El paralelismo entre la ontogenia y la filogenia ha llamado la atención de biólogos y filósofos por siglos ${ }^{7}$. Un icono de esta idea fue el biólogo y filósofo alemán Ernst Haeckel (1834-1919), quien a fines

${ }^{4}$ Los siete tans del Tangram corresponden a cinco triángulos de distinto tamaño, un cuadrado y un paralelógramo romboide.

${ }^{5}$ En la ontogenia, el individuo existe en el espacio físico y su identidad nunca se interrumpe durante el desarrollo. En la filogenia, la especie sólo existe en el dominio histórico y, a diferencia del individuo, no constituye un componente causal en la fenomenología evolutiva (Maturana y Varela, 1973).

${ }^{6}$ Los cambios de configuración estructural permitidos en este contexto son sólo aquellos que mantienen intacta la organización del ser vivo.

7 Una discusión extensa de este tópico se puede encontrar en Would 

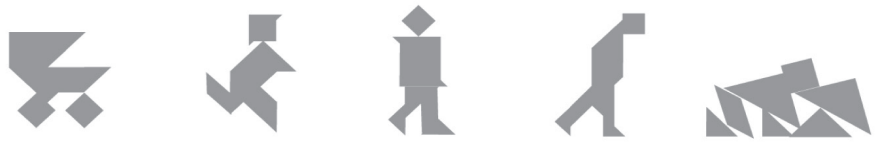

ONTOGENIA
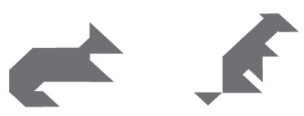

Origen

Expresión
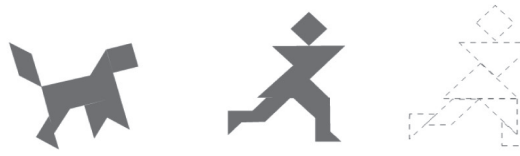

FILOGENIA

Alteración

Nota: La forma exhibe eventos de origen, expresión, transformación y alteración, tanto en la dimensión de la ontogenia como en la filogenia.

del siglo XIX propuso la teoría de la recapitulación ${ }^{8}$. En ella, la ontogenia de cada organismo, en particular su desarrollo embrionario, repite la historia evolutiva de la especie a la cual ese organismo pertenece (filogenia). Dicho de otra manera, cada etapa que el organismo atraviesa a lo largo de su desarrollo embrionario corresponde a una de las formas adultas que aparecieron en la historia evolutiva de la especie. Hoy en día la versión literal de esta teoría ha perdido seguidores, en gran parte por el surgimiento de la teoría sintética de la evolución ${ }^{9}$. Sin embargo, el interés formal por entender la relación íntima que se establece entre las dimensiones de la ontogenia y la filogenia ha dado origen recientemente a una nueva disciplina de la biología conocida como Evo-Devo ${ }^{10}$.

${ }^{8}$ Aun cuando los primeros en proponer esta teoría fueron John Hunter (1728-1793) y Carl Friedrich Kielmeyer (1795-1844), fue Ernst Haeckel quien la expuso de un modo sistemático.

${ }^{9}$ Síntesis de la teoría de la evolución de las especies por selección natural de Charles Darwin, la teoría genética de Gregor Mendel como base de la herencia biológica, la mutación genética aleatoria como fuente de variación y la genética de poblaciones matemática.

${ }^{10}$ Del inglés evolutionary developmental biology. Rama de la biología que compara el proceso de desarrollo de diferentes organismos con el fin de identificar los mecanismos del desarrollo que dan origen a cambios evolutivos en los fenotipos de los individuos. 
El entendimiento que la ciencia ha alcanzado sobre las bases genéticas del desarrollo embrionario desde una perspectiva comparada entre las especies nos permite comprender hoy que en el proceso evolutivo la filogenia actúa sobre los mismos principios biológicos sobre los cuales se basa la ontogenia, y por lo tanto sus restricciones obedecen a las mismas restricciones impuestas por ella. ¿Cuáles son estos mecanismos y restricciones? ¿Cuáles son las estrategias de la ontogenia para generar forma en los seres vivos?

\section{Origen de la forma en los seres vivos}

Para ilustrar las estrategias de la ontogenia en la generación de forma, emplearemos la analogía de la "caja negra"11 de la teoría de sistemas, enfocándonos en el grupo de seres vivos conocido como reino animal o metazoos ${ }^{12}$. En esta analogía, un organismo en etapas tempranas de su desarrollo atraviesa la caja negra de la ontogenia para dar origen a un individuo adulto (Figura $\mathrm{N}^{\circ} 2$ ). Dentro de esta caja negra operan dos estrategias fundamentales para generar forma, conocidas como determinística y regulativa. En todos los animales se dan ambas estrategias, en mayor o menor proporción, por lo que podemos considerar que ellas son dos caras de un mismo proceso.

Las estrategias determinística y regulativa pueden ser distinguidas una de la otra en forma experimental mediante el uso de técnicas embriológicas. La biología del desarrollo se ha preguntado qué ocurre con el organismo cuando una parte de él se remueve o se cambia de lugar (transplanta) en una etapa inicial de su ontogenia.

\subsection{Estrategia determinística, códigos en mosaico y el origen de la forma}

En la estrategia determinística, la remoción de una parte del organismo en desarrollo genera la pérdida de un segmento en el organismo adulto, mientras que el transplante resulta en un cambio de posición del

${ }^{11}$ Elemento que es estudiado desde el punto de vista de las entradas que recibe y las salidas o respuestas que produce.

12 Organismos cuyo cuerpo está constituido por muchísimas células diferenciadas y agrupadas en forma de tejidos, órganos y aparatos. Por ejemplo, los vertebrados, los moluscos y los gusanos (según Real Academia Española). 
FIGURA N ${ }^{\circ}$ 2: $\quad$ ANALOGÍA DE LA CAJA NEGRA DE LA TEORÍA DE SISTEMAS PARA ILUSTRAR LAS ESTRATEGIAS DETERMINÍSTICA (A, B) Y REGULATIVA (C, D) QUE SE DAN EN LA GENERACIÓN DE FORMA DURANTE LA ONTOGENIA DE LOS ANIMALES

a

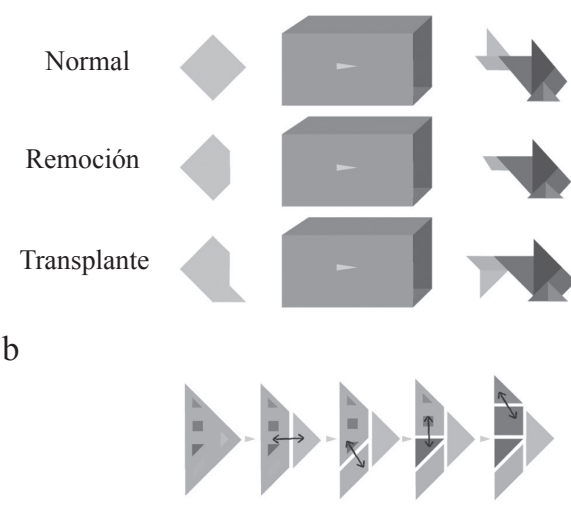

c

\section{Regulativa}

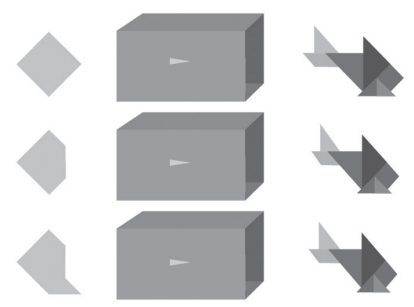

d

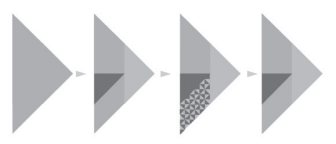

segmento transplantado. Esto último se puede observar en el ejemplo del desarrollo de la aleta posterior del pez de la Figura $N^{o} 2$ a. Al observar lo que ocurre dentro de la caja negra de la ontogenia, nos percatamos que al inicio del desarrollo el organismo contiene códigos de destino que se disponen formando un verdadero mosaico (Figura $\mathrm{N}^{\circ} 2 \mathrm{~b}$ ). A medida que el organismo se divide en sus distintos segmentos, éstos heredan códigos únicos que le darán identidad dentro del organismo adulto. La herencia de códigos predeterminados junto a una segregación espacial estereotipada asegura que la ontogenia sea invariable de individuo a individuo. Así, al remover un segmento del organismo se removerá a su vez una parte del código con la consecuente pérdida de información en el organismo adulto. De la misma forma, el transplante cambiará el ordenamiento de los códigos, generando un cambio de posición de segmentos definidos en el organismo adulto.

El desarrollo determinístico es característico de muchos animales invertebrados, siendo la lombriz Caenorhabditis elegans el caso de la literatura científica mejor descrito. Cabe destacar que la característica altamente determinística del desarrollo de C. elegans ha permitido dibujar la ruta que sigue cada una de las células durante la ontogenia, desde 
su origen y a través de todas las divisiones que las células experimentan hasta llegar a ser parte del organismo adulto, generando un verdadero árbol genealógico celular.

\subsection{Estrategia regulativa, organizadores y el origen de la forma}

En la estrategia regulativa tanto la remoción de un segmento como el transplante no afectan el resultado final del proceso ontogénico (Figura 2c). Esta propiedad de adaptarse a la interferencia tiene su origen en el mecanismo utilizado para otorgar información a cada uno de los segmentos del organismo en desarrollo. A diferencia de lo observado en la estrategia determinística, en la estrategia regulativa no existen códigos de destino organizados en mosaico dentro de la caja negra de la ontogenia (Figura 2d). En cambio, la información se va generando a partir de la interacción entre las partes. Por ello, la posición que cada segmento adopte dentro del organismo en desarrollo jugará un papel fundamental en su destino final, ya que condicionará el tipo de interacciones que podrá experimentar durante la ontogenia. En resumen, la forma no está pre-determinada en la estrategia regulativa sino que se genera a partir de la interacción de sus componentes. Esto permite que tras la manipulación experimental (remover o transplantar un segmento) el sistema sea capaz de adaptarse y recuperar su condición inicial, siguiendo un curso normal de desarrollo ontogénico. Este tipo de desarrollo es característico de los animales vertebrados ${ }^{13}$, dentro de los cuales nos encontramos los seres humanos.

La característica central de la estrategia regulativa, es decir, la capacidad de generar forma a través de la interacción de sus componentes, fue demostrada experimentalmente en embriones de anfibios por el científico alemán Hans Spemann (1869-1941) y su esposa Hilde Mangold, en estudios pioneros que permitieron la obtención del Premio Nobel de Medicina en el año $1935^{14}$.

${ }^{13}$ Animales cordados que tienen esqueleto con columna vertebral y cráneo, y sistema nervioso central constituido por médula espinal y encéfalo (Real Academia Española).

${ }^{14}$ Hans Spemann fue un embriólogo experimental y maestro de técnicas de microcirugía en huevos de anfibio que estudió medicina en la Universidad de Heidelberg y zoología en el Instituto Zoológico de la Universidad de Würzburg. Sus estudios fundaron la teoría de inducción embrionaria por organizadores embrionarios, permitiendo el desarrollo de la ciencia moderna de morfogénesis experimental. 
Los anfibios, al igual que otros animales vertebrados, se desarrollan a partir de una célula única. En los estadios iniciales, esta célula se divide rápidamente hasta dar origen a un embrión con miles de células más pequeñas de apariencia semejante, el cual sufre cambios dramáticos de forma que le confieren un aspecto alargado, con una cabeza, tronco y cola claramente definidos. Spemann y Mangold evidenciaron que una región discreta del embrión temprano de anfibio exhibe los primeros cambios de forma y coincide espacialmente con aquella zona donde más tarde se formará el eje central del embrión. Al transplantar esta región, denominada "labio dorsal del blastoporo", hacia una región aún no diferenciada de otro embrión de características similares, Spemann y Mangold observaron la formación de un embrión secundario unido al embrión principal. El hallazgo principal de ambos investigadores fue percatarse que el embrión secundario contenía células provenientes de la zona que recibía el transplante y no del transplante en sí mismo. Es decir, este simple experimento demostraba que el transplante era capaz de producir señales que instruían a la zona vecina que lo albergaba a cambiar su destino original y formar un embrión nuevo. El descubrimiento de esta región inductora o centro organizador sentó las bases para el entendimiento del proceso central de la estrategia regulativa, conocido como inducción celular, mediante la cual un grupo de células del embrión puede cambiar el destino de otras células.

Hoy se entiende que existen distintos centros organizadores que participan durante el desarrollo y que la actividad coordinada de ellos es finalmente responsable de generar la forma característica del embrión. Estos centros organizadores producen señales moleculares que son capaces de difundir, incluso alcanzando largas distancias, generando respuestas discretas en las células que las reciben, las que muchas veces dependen de la concentración de la señal que difunde. En ese último caso, las señales se denominan morfógenos. El biólogo inglés Lewis Wolpert ${ }^{15}$ utilizó la bandera francesa para ilustrar cómo a partir de un número reducido de señales de tipo morfógeno se pueden generar patrones más o menos complejos. En su ejemplo, un morfógeno es producido por un centro organizador, difunde y genera una gradiente decre-

15 Académico e investigador de biología aplicada a la medicina en el Departamento de Anatomía y Biología del Desarrollo del University College de Londres. Ha publicado, entro otros libros, The Triumph of the Embryo (1991) y Principles of Development (1998). 
ciente de concentración. Aquellas células que reciben concentraciones altas del morfógeno responden adquiriendo un color azul, aquellas que leen concentraciones intermedias se vuelven blancas, mientras que las que reciben bajas concentraciones se tornan rojas. En su conjunto, la combinación de los tres tipos de respuesta genera un patrón espacial de bandera francesa.

\subsection{Combinando las estrategias determinística y regulativa en la ontogenia}

Hemos descrito que existen dos estrategias fundamentales en el origen de forma, y que todos los animales las usan en mayor o menor proporción. Pero ¿cómo se combinan ambas estrategias en un organismo en desarrollo? Para ilustrar este punto utilizaremos la secuencia de Tangram de la Figura $\mathrm{N}^{\mathrm{o}} 3$. Extraeremos un segmento del organismo en distintas etapas de su ontogenia, y nos preguntaremos qué es capaz de originar este segmento si se desarrolla en un ambiente aislado del organismo que le dio origen.

Podemos observar que un segmento extraído en etapas muy tempranas de la ontogenia es capaz de generar un organismo completo (totipotencialidad) y que, a medida que transcurre la ontogenia, esta capacidad se va perdiendo gradualmente, pudiendo dar origen en una primera instancia a todos los segmentos pero no al organismo completo (pluripotencialidad), luego sólo a parte de los segmentos (oligopotencialidad) para finalmente ser capaz de originarse solo a sí mismo (unipotencialidad). Este comportamiento es característico de la gran mayoría de los animales vertebrados, incluyendo a los seres humanos. Por lo tanto, el inicio de la ontogenia es por naturaleza regulativo, donde sus componentes exhiben una alta potencialidad, y por lo tanto poseen un muy bajo compromiso a dirigirse hacia un destino en particular. A medida que la ontogenia avanza, la potencialidad se va perdiendo y el compromiso va siendo cada vez mayor, por lo que la estrategia determinística cobra una mayor relevancia. En un organismo adulto tenemos una combinación de ambos tipos de estrategias. Por un lado, todos los tejido diferenciados de nuestros órganos, como el cerebro, los huesos y la piel, tienen un alto compromiso y eso permite mantener su organización morfológica y funcional. Sin embargo, al mismo tiempo, en casi todos nuestros tejidos, con algunas excepciones como el sistema nervioso, existe una continua regeneración de los componentes y por lo 

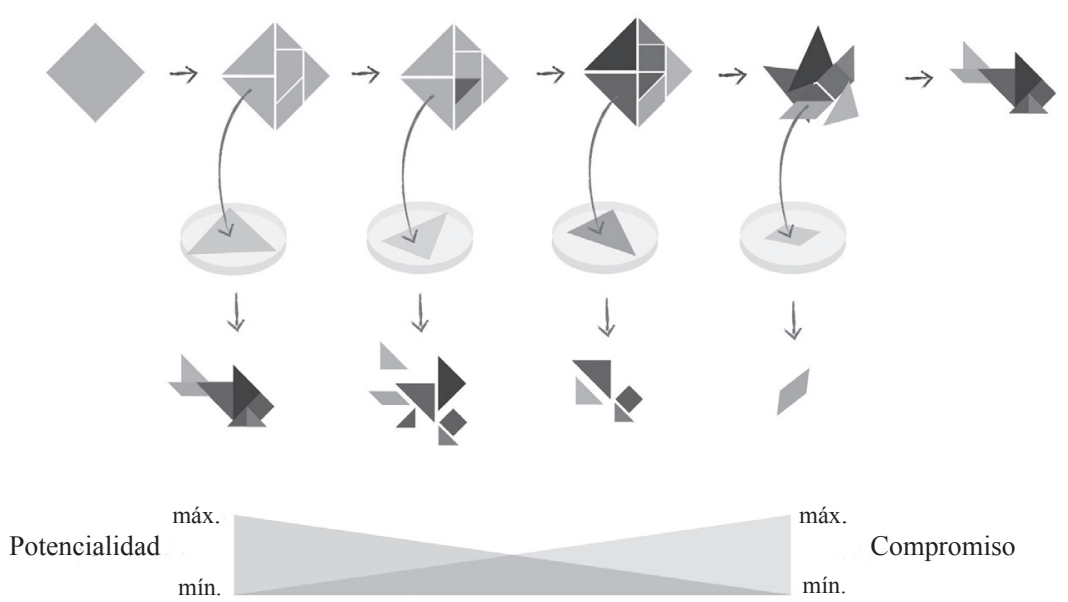

tanto en estos tejidos habitan elementos con una alta potencialidad que permiten organizar esta regeneración. Estos elementos se conocen como células madre.

Estudios de las últimas décadas han demostrado que el "origen" de la forma a través de las estrategias determinística y regulativa depende en gran medida de la acción de factores genéticos y de su interacción con el medio ambiente durante la ontogenia. Uno de los ejemplos más demostrativos de este concepto proviene de los experimentos de manipulación genética realizados en la mosca Drosophila melanogaster. En ellos se demostró que al afectar la función de genes específicos se podía alterar la generación de forma en el embrión, y que este efecto podía ser extenso o discreto de acuerdo a la función del gen dentro de una escala jerárquica de actividad. Por ejemplo, los genes denominados "maestros" ocupaban la posición más alta en la jerarquía y coordinaban globalmente la función de muchos otros genes, los que a su vez modulaban la acción de otros factores genéticos en lugares más restringidos del embrión. Al final de esta jerarquía se encontraban los genes de la morfogénesis, que son los responsables directos de los cambios de forma y, por lo tanto, permiten que la forma se "exprese" durante la ontogenia. Los mecanismos que estos genes emplean para 
expresar la forma en los seres vivos aún no se entienden a cabalidad, sin embargo, se sabe que sus efectos se rigen de acuerdo a las leyes de las matemáticas y la física.

\section{Expresión de la forma en los seres vivos}

El biólogo y matemático escocés Sir D'Arcy Wentworth Thompson (1860-1948) fue pionero de la matemática biológica y publicó en 1917 su libro seminal titulado On Growth and Form, el cual ha servido de inspiración para muchas generaciones de biólogos y matemáticos, y en particular para quienes trabajan en las fronteras de las disciplinas. En este libro, Thompson explora de manera descriptiva y sin ahondar en explicaciones mecanicistas, el posible papel que juegan las matemáticas y la física en la determinación de la forma y la estructura de los seres vivos. En una de sus secciones más difundidas denominada "Sobre la teoría de la transformación, o la comparación de formas relacionadas", Thompson mostró que las diferencias de forma entre especies de animales relacionados pueden explicarse por medio de transformaciones geométricas relativamente simples. Por ejemplo, dos especies de peces submarinos de la familia Sternoptychidae, que en lo superficial parecen muy diferentes, pueden ser transformadas una en la otra mediante transformaciones cartesianas (Figura $\mathrm{N}^{\circ} 4$ ). A partir de esta observación y numerosas otras, dentro de las que destacan la comparación de la espiral de la concha de distintos caracoles, Thompson sugiere que el cambio evolutivo producido entre especies sería el resultado de cambios en la gradiente, velocidad o el tiempo de desarrollo, los que generarían cambios de los espacios morfológicos de acuerdo a principios físicos y matemáticos.

Los estudios de Thompson se reconocen como pioneros en establecer una relación entre la biología, las matemáticas y la física, ya que en su época no existían métodos para inferir o medir directamente las fuerzas mecánicas involucradas en los procesos de generación de forma. Hoy en día es posible visualizar los cambios de forma durante la ontogenia en un organismo completo, y en muchos casos inferir, o directamente medir, las fuerzas mecánicas que gobiernan los distintos procesos generadores de forma. Por medio de estas aproximaciones se ha comprobado que fenómenos físicos, en particular del campo de la 

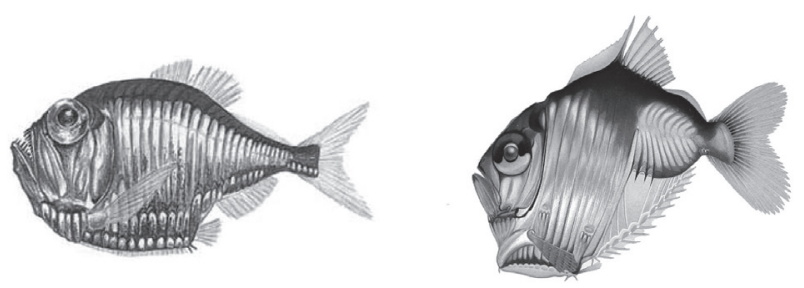

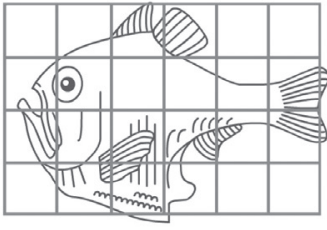

Argyropelecus olfersi

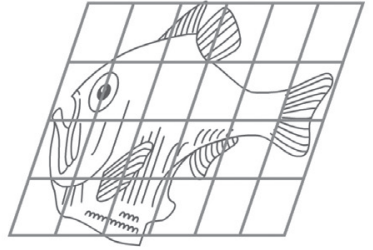

Sternoptyx diaphana

Nota: La deformación de una malla cartesiana impuesta sobre la especie Argyropelecus, la cual demarca un denominado espacio morfométrico, cambia la forma externa de esta especie en aquella de la especie Sternoptyx. (Ilustración modificada de Thompson, 1942)

mecánica ${ }^{16}$, juegan un papel central en la expresión de la forma resultante de la ontogenia. ¿Cómo se manifiestan esos fenómenos mecánicos en la morfogénesis de un animal?

Para responder esta pregunta nos valdremos de uno de los peces submarinos utilizados por Thompson, y realizaremos un viaje virtual hacia la conformación cartesiana inicial de su ontogenia (Figura $\mathrm{N}^{0} 5 \mathrm{a}$ ). Este viaje hipotético nos sirve de analogía para resaltar tres conceptos centrales del proceso de expresión de forma biológica. Primero, que la conformación cartesiana inicial de la ontogenia contiene unidades primordiales sobre las cuales actúan las estrategias determinística y regulativa para permitir la expresión y transformación de la forma. Estas unidades morfogenéticas están conformadas por células, las que actúan como verdaderos ladrillos generadores de forma. Segundo, que al comienzo del proceso generador de forma no existe una representación

${ }^{16}$ Rama de la física que trata del equilibrio y del movimiento de los cuerpos sometidos a cualquier fuerza (Real Academia Española). 
en miniatura del resultado final de la ontogenia, sino que la forma final aparece como resultado de cambios progresivos que experimentan las unidades morfogenéticas durante el proceso de desarrollo. Y tercero, que los cambios de forma que se originan en cada una de las unidades morfogenéticas se transmiten a niveles de organización biológica más complejos como tejidos y órganos, para de esta manera moldear los cambios de forma del organismo completo (Figura $\mathrm{N}^{\circ} 5 \mathrm{a}$ ).

Los principios que gobiernan la expresión de forma en las unidades morfogenéticas primordiales del embrión se basan en el entendimiento que las células se pueden comportar de dos maneras distintas durante la ontogenia. Por un lado, pueden organizarse como grupos y formar tejidos, expresando los cambios de forma de manera coordinada,

a

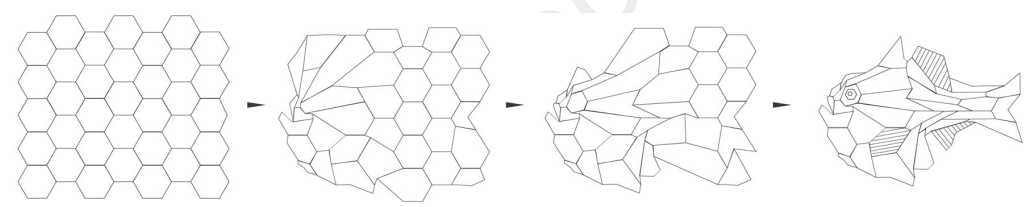

b

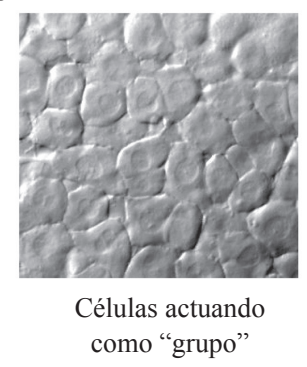

$\mathrm{c}$

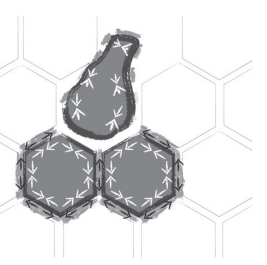

Tensión cortical

VS

d

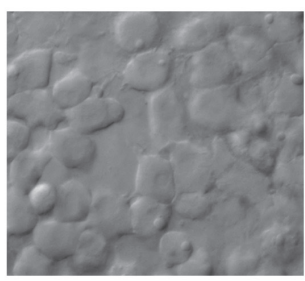

Células actuando como "individuos"

adhesión

Nota: (a) Viaje virtual entre la configuración cartesiana inicial y final de Argyropelecus olfersi. En el arreglo inicial (izquierda), cada uno de los hexágonos representa una unidad morfogenética primordial que durante la ontogenia sufre transformaciones que impactan la forma del organismo completo. (b-d) En las unidades morfogenéticas primordiales, las células exhiben dos tipos de comportamiento, como grupo (b) y como individuos (d). En ambos casos, los cambios de forma que ellas experimentan son el resultado del balance existente entre las fuerzas mecánicas de tensión (flechas blancas) y adhesión (flechas negras) (c). 
como un todo. Este comportamiento contrasta con el otro tipo en el cual la célula se comporta como un individuo, cambiando de forma y desplazándose de manera autónoma con respecto a su entorno. Durante la ontogenia, una misma célula o unidad morfogenética primordial puede transitar por ambos tipos de comportamiento en distintas etapas, y de ello dependerá su contribución a la expresión de forma en el organismo. En ambos tipos de comportamiento, los cambios de forma que las células experimentan se pueden explicar por fenómenos de tipo físico/ mecánico. La forma que una célula adopta es el resultado del balance de dos fuerzas contrapuestas. Una de ellas es la tensión cortical, que depende de una malla contráctil que se encuentra en la corteza de la célula y cuya contracción tiende a hacer que la célula adopte una forma esférica. La otra es la fuerza de adhesión de la célula con su entorno vecino, que pueden ser otras células o una matriz acelular, la que hace que la célula adopte formas más complejas. Así, aquellas células que muestran un balance de fuerzas en favor de una adhesión estable con su entorno, forman agrupaciones compactas tipo tejidos y adoptan formas poligonales. Por otro lado, las células que establecen adhesiones más inestables y que muestran gran dinámica en su tensión cortical son capaces de adquirir formas más redondeadas y cambiantes, y separarse de los tejidos para adoptar un comportamiento migratorio. En este contexto, el papel que juegan las estrategias determinística y regulativa, las que están bajo el control de factores genéticos, será finalmente modular el balance entre tensión y adhesión en las distintas unidades morfogenéticas de la ontogenia, y asimismo coordinar la transmisión de estos fenómenos mecánicos a niveles más complejos de organización biológica, impactando la forma del organismo completo. La simpleza de esta idea, que hoy cautiva a embriólogos experimentales, hace honor al postulado original de Sir D'Arcy Wentworth Thompson sobre el papel de las matemáticas y la física en la morfogénesis biológica:

"Células y tejidos, conchas y hueso, hojas y flores, son tantas porciones de la materia, y es en obediencia a las leyes de la física que sus partículas se han movido, moldeado y conformado".

On Growth and Form (1917) 


\section{REFERENCIAS BIBLIOGRÁFICAS}

Arnaout, Rima y Didier Stainier. "Developmental Biology: Physics Adds a Twist to Gut Looping”. Current Biology, Vol. 21, pp. R854-R857, 2011. Camazine, Scott, Jean-Louis Deneubourg, Nigel Franks, James Sneyd, Guy Theraulaz y Eric Bonabeu. "Self-Organization in Biology". Princeton University Press, 2001.

Gilber, Scott y Anne Raunio. Embryology: Constructing the Organism. Sinauer Associates, Inc. Publishers, 1997.

Hutson, Shane y Xiaoyan Ma. Mechanical Aspects of Developmental Biology:

Perspectives On Growth and Form in the (Post)-Genomic Age. IOP Publishing, Phys. Biol, Vol. 5, pp. 015001, 2008.

Lawrence, Peter y Michael Levine: "Primer: Mosaic and Regulative Development: Two Faces of One Coin". Current Biology, Vol. 16, pp. R236-R239, 2006.

Maturana, Humberto y Francisco Varela. De Máquinas y Seres Vivos. Autopoiesis: La Organización de lo Vivo. Editorial Universitaria, 1973.

Poughg, Harvey. Vertebrate Life. Prentice Hall, Fourth Edition, 1996.

Thompson, D'Arcy Wentworth. On Growth and Form (a new edition). Cambridge University Press, 1942.

Wolpert, Lewis. Principles of Development. Oxford University Press, second edition, 2002.

Would, Stephen Jay. Ontogeny and Phylogeny. Harvard University Press, 1977. 